\title{
Clinical evaluation of haploidentical hematopoietic combined with human umbilical cord-derived mesenchymal stem cells in severe aplastic anemia
}

\author{
Lixin $\mathrm{Xu}^{1 \dagger}$, Zhouyang Liu ${ }^{1 \dagger}$, Yamei $\mathrm{Wu}^{1}$, Xueliang Yang ${ }^{1}$, Yongbin Cao ${ }^{1}$, Xiaohong Li ${ }^{1}$, Bei Yan ${ }^{1}$, Songwei Li ${ }^{1}$, \\ Wanming $\mathrm{Da}^{2}$ and Xiaoxiong $\mathrm{Wu}^{1^{*}}$
}

\begin{abstract}
Background: This study not only evaluated the clinical effects of treatment using haploidentical hematopoietic stem cells (haplo-HSCs) combined with human umbilical cord mesenchymal stem cells (UC-MSCs) in patients with severe aplastic anemia (SAA), but also investigated the factors related to graft versus host disease (GVHD).

Methods: Cotransplantation of haplo-HSCs and UC-MSCs was performed in 24 SAA patients. The conditioning regimens consisted of rabbit anti-human T-lymphocyte immunoglobulin (ATG), cyclophosphamide, and fludarabine with or without busulfan. GVHD was prevented using cyclosporine A, ATG, anti-CD25 monoclonal antibody, and mycophenolate material.

Results: The incidence of acute GVHD was 50\%. The incidence of severe acute GVHD was not related to gender, age, donor-recipient relations, and patient/donor pair, while patient/donor pair $(r=0.541, P=0.022)$ was significantly correlated with incidence of chronic GVHD. Upon follow-up for a median of 13 months, 5 of the 24 patients (20.8\%) were dead. The survival rates at 3 and 6 months in all patients were $87.5 \%$ (21/24) and 83.3\% (20/24), respectively.
\end{abstract}

Conclusion: Cotransplantation of haplo-HSCs combined with UC-MSCs was an effective and safe approach for the treatment of patients with SAA. The appropriate conditioning regimen and early treatment for infection also played a critical role in the success of HSCT.

Keywords: Severe aplastic anemia, Haploidentical hematopoietic stem cells transplantation, Umbilical cord-derived mesenchymal stem cells, Graft versus host disease

\section{Background}

Severe aplastic anemia (SAA) is a life-threatening disease characterized by hypoplastic bone marrow and pancytopenia [1]. Although allogeneic hematopoietic stem cell transplantation (HSCT) is the first choice for the treatment of SAA [2], it is difficult to search for the human leukocyte antigen (HLA)-compatible donors in China [3].

\footnotetext{
*Correspondence: xiongwuxiao@suhu.com

'Lixin $\mathrm{Xu}$ and Zhouyang Liu are joint first authors

${ }^{1}$ Department of Hematology, The First Affiliated Hospital of Chinese PLA

General Hospital, No. 51 Fucheng Road, Haidian District, Beijing 100048,

China

Full list of author information is available at the end of the article
}

Recent studies have investigated the effect of haploidentical HSCT (haplo-HSCT) in SAA patients, and it has been considered as an optional treatment [4-7]. Nevertheless, the high incidence of graft versus host disease (GVHD) limited the clinical application of haplo-HSCT in patients with SAA.

It has been demonstrated that mesenchymal stem cells (MSCs) can reduce the risks of both acute GVHD (aGVHD) and chronic GVHD (cGVHD) [8]. Notably, human umbilical cord-derived mesenchymal stem cells (UC-MSCs) have higher activities of proliferation and differentiation in comparison with the bone marrow MSCs [9]. Recent study has revealed that UC-MSCs can 
not only reduce the risk of GVHD, but also increase the transplantation rate of allogeneic HSCs [10]. A previous study by $\mathrm{Wu}$ et al. [11] and another research by Zhang et al. [12] have preliminarily shown that the cotransplantation of haplo-HSCs and third-party donor-derived UC-MSCs achieved favorable outcomes for patients with aplastic anemia. However, several problems remain to be further resolved, such as the correlation factors affecting GVHD and lack of optimal sources of donors.

In this study, we reviewed 24 patients with SAA who received the cotransplantation of haplo-HSCs and UCMSCs with modified conditioning, and evaluated the safety and efficacy of the cotransplantation of UC-MSCs and donor HSCs in SAA patients and investigated the factors related to GVHD.

\section{Methods}

\section{Patients}

The whole protocol was approved by the Institutional Review Board of our hospital, and informed written consents were obtained from all patients. All donors were eligible for donating HSCs, and they also signed the informed consent forms for donation before transplantation.

This study recruited 24 patients with SAA (14 males and 10 females) who underwent cotransplantation of human UC-MSCs and HSCs from June 2010 to August 2013. The inclusion criteria were as follows: patients presenting with SAA or very severe aplastic anemia (VSAA) were defined according to the International Aplastic Anemia Study Group [13]; patients who underwent previous therapy regimens, including cyclosporine A (CSA), stanozole/andriol, granulocyte colony stimulating factor (G-CSF), anti-human T-lymphocyte immunoglobulin (ATG), Erythropoietin (EPO), glucocorticoid-however, they failed to respond to this therapy regimen; patients who received multiple transfusions and whose transfusion dependence was confirmed when the transplantation was performed; patients who agreed to participate in HSCT; and all donors who were relatives to the patients. Patients who had uncontrolled infections and severe liver, renal, lung, or heart diseases before transplantation were excluded in this study. The detailed information about patients and preparation of donors is listed in Additional file 1: Table S1.

\section{Preparation of UC-MSCs and HSCs}

UC-MSCs were purchased from the National Engineering Research Center of Cell Products. HSCs were isolated from peripheral blood of the donors who were injected subcutaneously with recombinant human G-CSF (rhGCSF, $5 \mu \mathrm{g} / \mathrm{kg}$ day) for 5-6 consecutive days. In brief, peripheral blood (200-600 mL) was collected on day 5, and red cells were removed to avoid the incompatibility of major cross-match. Then, HSCs were separated using Fenwal CS-3000 plus blood cell separator (Baxter International Inc., Deerfield, IL, USA) on days 6 and 7. Finally, mononuclear cells $\left[(4-6) \times 10^{8} / \mathrm{kg}\right]$ and $\mathrm{CD} 34^{+}$cells $[(2-$ 4) $\times 10^{8} / \mathrm{kg}$ ] were obtained.

\section{HSCs and UC-MSCs transplantation}

Anti-inflammatory, hepatoprotective and gastric mucosa-protecting treatments were performed on patients with sodium bicarbonate, dexamethasone and promethazine before transplantation. The conditioning regimens included rabbit ATG (Fresenius AG, Oberursel, Germany), cyclophosphamide (Cy), and fludarabine (Flu) treatment. For the patients with acute SAA (SAAI), intravenous administration of $30 \mathrm{mg} /\left(\mathrm{m}^{2}\right.$ day) of Flu and $500-800 \mathrm{mg} /\left(\mathrm{m}^{2}\right.$ day) of Cy was performed from days -5 to -2 , and $5 \mu \mathrm{g} /(\mathrm{kg}$ day) of ATG was administered from days -4 to -1 (Fig. 1a). For the patients with chronic SAA (SAA-II), the same treatment of ATG and Cy was applied as above with the supplement of $0.6 \mathrm{mg} /$ ( $\mathrm{kg} 6 \mathrm{~h}$ ) of busulfan (BU) from days -8 to -5 prior to transplantation (Fig. 1b) [3]. On day 0, HSCs were infused intravenously. Then, a total of $5 \times 10^{5} / \mathrm{kg}$ UC-MSCs was transfused at $4 \mathrm{~h}$ before infusion with HSCs.

\section{Management of GVHD}

Prophylaxis of GVHD was carried out by the administration of immunosuppressive agents, such as CSA, mycophenolate mofetil (MMF), and anti-CD25 antibody (CD25Ab; Basiliximab, Novartis Pharma Stein AG, Stein, Switzerland). To achieve a target trough with blood concentration of $250-450 \mathrm{ng} / \mathrm{mL}$ at 12 months after HSCT, CSA ( $3 \mathrm{mg} / \mathrm{kg}$ ) was conducted by intravenous injection from day -5 (Fig. 1); then, CSA concentration was gradually decreased and withdrawn in the next 2-3 months. Oral MMF administration ( $20 \mathrm{mg} / \mathrm{kg} /$ day) was conducted from days -1 to day +100 , and intravenous injection of CD25Ab monoclonal antibody $(0.5 \mathrm{mg} / \mathrm{kg} /$ day $)$ was performed only on day +4 after HSCT (Fig. 1). GlucksbergSeattle criteria (GSC) [14] were used for the diagnosis and grading of GVHD, and those with grade III-IV acute GVHD (aGVHD) were served as severe aGVHD.

\section{Supportive care}

Patients were housed in the laminar flow clean ward. They were asked to take a medicated bath, and treated with acyclovir, ornidazole, sulfamethoxazole trimethoprim, and sulfadiazine 1 or 2 days before conditioning. On day +3 after HSCT, G-CSF and thrombopoietin (TPO) were administered subcutaneously until the achievement of hematopoietic reconstitution. Prostaglandin E1 (PGE1; $20 \mu \mathrm{g} /$ day; Beijing Tide Pharmaceutical Co., LTD, Beijing, 


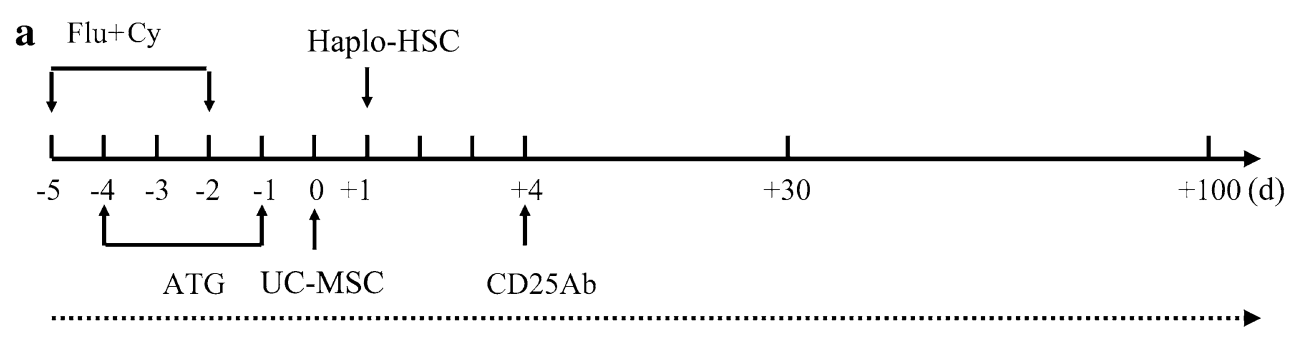

$\mathrm{MMF}+\mathrm{CSA}$

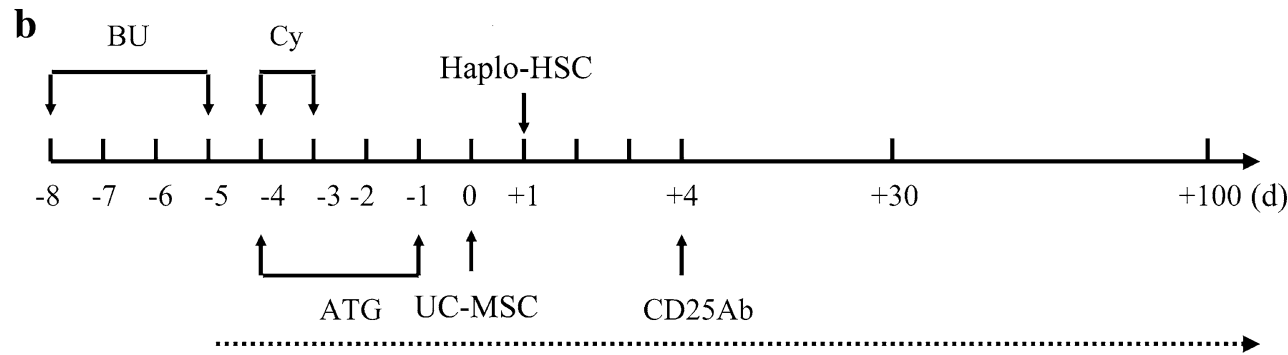

$\mathrm{MMF}+\mathrm{CSA}$

Fig. 1 The schematic diagram of conditioning regimen and prophylaxis and management of GVHD. Conditioning included 1 of 2 regimens: for patients with SAA-I (a), intravenous administration of fludarabine (Flu) $30 \mathrm{mg} /\left(\mathrm{m}^{2}\right.$ day) and cyclophosphamide (Cy) $500-800 \mathrm{mg} /\left(\mathrm{m}^{2} \mathrm{day}\right) \mathrm{from}$ day -5 to -2 , anti-human T-lymphocyte immunoglobulin (ATG) $5 \mu \mathrm{g} /(\mathrm{kg}$ day) from day -4 to -1 ; or for SAA-II (b), the same treatment of ATG and Cy with busulfan (Bu) $0.6 \mathrm{mg} /(\mathrm{kg} 6 \mathrm{~h}$ ) from day -7 to -6 . Prophylaxis and management of GVHD included: intravenous administration of cyclosporine A (CSA) $3 \mathrm{mg} / \mathrm{kg}$ from day - 5, followed by gradual decrease in concentration after reaching a target trough blood concentration of 250-450 ng/mL and then withdrawal in the next 2-3 months, oral mycophenolate mofetil (MMF) administration (20 mg/kg/day) from day - 1 to +100 , and intravenous anti-CD25 antibody (CD25Ab) monoclonal antibody ( $0.5 \mathrm{mg} / \mathrm{kg} /$ day) on day +4 after HSCT. SAA-l: acute severe aplastic anemia, SAA-II: chronic severe aplastic anemia

China) and compound Danshen $(20 \mathrm{mg} /$ day; Shanghai No. 1 Biochemical \& Pharmaceutical Co., LTD, Shanghai, China) were administered from day -5 to day +14 as hepatic veno-occlusive disease (VOD) prophylaxis. Mesna injection (Baxter, Shanghai, China) was used to prevent the hemorrhagic cystitis (HC).

\section{Engraftment}

Neutrophil engraftment was defined as the first of three consecutive days with an absolute neutrophil count (ANC) above $0.5 \times 10^{9} / \mathrm{L}$, and platelet engraftment was defined as the first day of a week with the platelet count exceeding $20 \times 10^{9} / \mathrm{L}$ in the absence of transfusion. Hematopoietic chimerism was assessed using peripheral blood samples of the donor and recipient via short-tandem repeated sequence-PCR (STR-PCR) DNA fingerprinting [15] for sex-matched pairs and karyotype analysis for sex-mismatched pairs. The bone marrow of the recipient was analyzed for hematopoietic chimerism every 30 days until 90 days after HSCT. For blood-type incompatible pairs, the measurement of blood-type titer was processed weekly after hematopoietic reconstitution.

\section{Statistical analysis}

Patients were followed up for 3-44 months. KaplanMeier analysis was calculated as survival in the absence of death. The relationships between the incidence of GVHD and gender, age, blood type, donor-recipient relations, and patient/donor pair were evaluated using Chi-square test. All statistical analyses were performed using the standard statistical package of SPSS 19.0 (IBM, Armonk, New York, USA). A two-sided $P$ value of 0.05 or less was considered statistically significant.

\section{Results}

\section{Engraftment and chimerism}

Clinical features of patients and their donors, and the subsequent outcomes in 24 case are shown in Table 1. The median scores of infusion numbers of MNCs and $\mathrm{CD}^{+} 4^{+}$cells were $10.6 \times 10^{8} / \mathrm{kg}$ and $4.55 \times 10^{6} / \mathrm{kg}$ for all the patients, respectively. The median times to achieve neutrophil and platelet engraftment were 11 and 13 days, respectively. All the patients achieved $70-100 \%$ donor chimerism within 1 month after haplo-HSCT. 


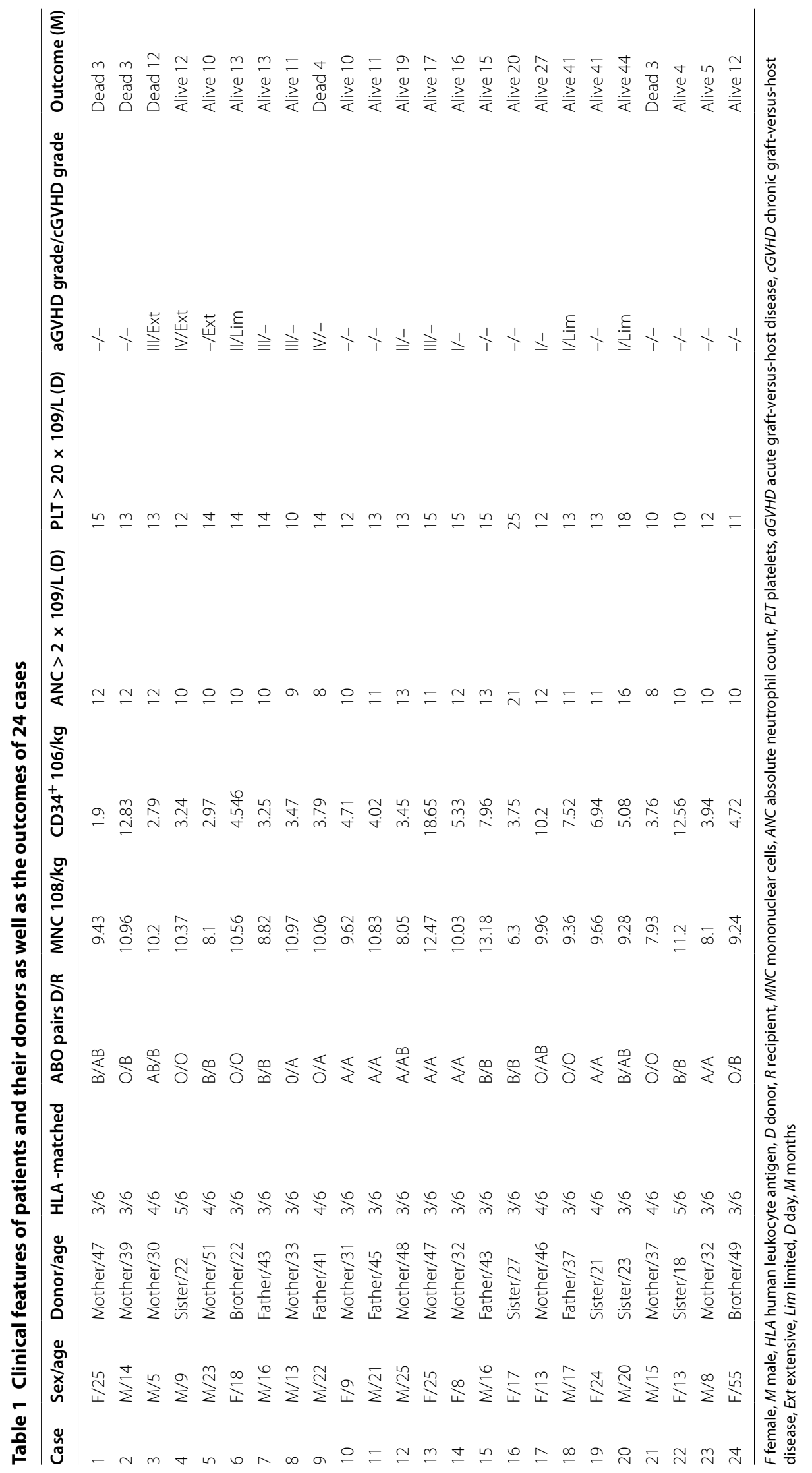




\section{GVHD}

The GVHD incidences of all patients after HSCT are shown in Table 2. Of the 24 patients, 12 cases $(50 \%)$ developed aGVHD, including 4 (16.7\%) with grade I, $2(8.35 \%)$ with grade II, 4 (16.7\%) with grade III, and 2 (8.35\%) with grade IV. Meanwhile, 3 of the 24 (12.5\%) patients developed extensive cGVHD, and 2 cases among them also experienced III-IV aGVHD. In addition, 3 of the $24(12.5 \%)$ patients who experienced I-II aGVHD also developed limited cGVHD. The other 11 patients had no GVHD. As shown in Table 3, the incidence of severe aGVHD was not related to gender, age, donorrecipient relations and patient/donor pair, while patient/ donor pair was significantly correlated with extensive

Table 2 GVHD incidences in allogeneic HSCT recipients

\begin{tabular}{ll}
\hline Parameters & $\boldsymbol{N = \mathbf { 2 4 } , \mathbf { ( \% ) }}$ \\
\hline Acute GVHD & \\
I & $4(16.7 \%)$ \\
II & $2(8.35 \%)$ \\
III & $4(16.7 \%)$ \\
IV & $2(8.35 \%)$ \\
Chronic GVHD & \\
Lim & $3(12.5 \%)$ \\
Ext & $3(12.5 \%)$
\end{tabular}

HSCT hematopoietic stem cell transplantation, aGVHD acute graft-versus-host disease, CGVHD chronic graft-versus-host disease, Ext extensive, Lim limited
cGVHD $(r=0.541, P=0.022)$. The postoperative incidences of extensive cGVHD were $2 / 15$ of patients with blood-type compatibility, 1/1 of major cross-match mismatch and 0 of minor cross-match mismatch. The rise in GVHD incidence paralleled the increase in HLA loci $\left(x^{2}=7.764, P=0.022\right)$. Extensive cGVHD trended to occur in male and patients who also suffered from severe aGVHD $(P=0.060$ and $P=0.099$, respectively).

\section{Other complications}

During HSCT period, all patients suffered from nausea, vomiting, and various degrees of anepithymia, and then these symptoms regressed after symptomatic and supportive therapies. Nineteen patients $(19 / 24,79.2 \%)$ who developed stomatitis also showed improvement after mouth care and the topical application of epithelium growth factor. At the stage of bone marrow suppression, various degrees of fevers occurred in patients, and then fevers were controlled by the administrations of imipenem, vancomycin, and antifungals. The incidence of pulmonary infection was $16.7 \%(4 / 24)$. Two cases with pulmonary infection developed into severe pneumonia, and one of them finally died. There were two patients with septicemia and two cases with engraftment syndrome. Fifteen patients $(15 / 24,62.5 \%)$ had viral infections, including Cytomegalovirus (CMV) or Epstein Barr Virus (EBV)-emia in eight cases, and two or more viruses infection in five cases. Three patients $(3 / 24,12.5 \%)$ developed HC. Sixteen patients suffered from diarrhea, and

Table 3 The correlation of variables with GVHD incidence in allogeneic HSCT recipients

\begin{tabular}{|c|c|c|c|c|c|c|}
\hline \multirow[t]{2}{*}{ Variables } & \multicolumn{3}{|l|}{ Severe aGVHD } & \multicolumn{3}{|c|}{ Extensive cGVHD } \\
\hline & Incidence (\%) & $r$ & $P$ value & Incidence (\%) & $r$ & $P$ value \\
\hline Gender & & 0.293 & 0.134 & & 0.319 & 0.060 \\
\hline Male & $5 / 14(35.7 \%)$ & & & $3 / 14(21.4 \%)$ & & \\
\hline Female & $1 / 10(10 \%)$ & & & $0 / 10$ & & \\
\hline Age, years & & 0.05 & 0.807 & & 0.033 & 0.872 \\
\hline$\geq 20$ & $2 / 9(22.2 \%)$ & & & $1 / 9(11.1 \%)$ & & \\
\hline$<20$ & $4 / 15(26.7 \%)$ & & & $2 / 15(13.3 \%)$ & & \\
\hline Donor-recipient relationship & & 0.083 & 0.599 & & 0.055 & 0.465 \\
\hline Mother-child & $3 / 12(25 \%)$ & & & $2 / 12(16.7 \%)$ & & \\
\hline Father-child & $2 / 5(25 \%)$ & & & $0 / 5$ & & \\
\hline Siblings & $1 / 7(14.3 \%)$ & & & $1 / 7(14.3 \%)$ & & \\
\hline Patient/donor pair & & 0.225 & 0.566 & & 0.541 & 0.022 \\
\hline 3 HLA loci & $3 / 16(18.8 \%)$ & & & $0 / 16$ & & \\
\hline 2 HLA loci & $2 / 6(33.3 \%)$ & & & $2 / 6(33.3 \%)$ & & \\
\hline 1 HLA loci & $1 / 2(50 \%)$ & & & $1 / 2(50 \%)$ & & \\
\hline Severe aGVHD & & & & & 0.364 & 0.099 \\
\hline Yes & & & & $2 / 6(33.3 \%)$ & & \\
\hline No & & & & $1 / 18(5.6 \%)$ & & \\
\hline
\end{tabular}

HSCT hematopoietic stem cell transplantation, aGVHD acute graft-versus-host disease, CGVHD chronic graft-versus-host disease, HLA human leukocyte antigen 
they all responded well to antidiarrheal treatment and regulation of intestinal microecology. The incidences of infectious diarrhea, fungal enteritis, and viral enteritis were $29.2 \%$ (7/24), $4.2 \%(1 / 24), 16.7 \%$ (6/24), respectively, and all of these cases developed into grade III-IV intestinal GVHD. Besides, three patients developed epilepsy. Among them, seizure was resolved in one patient since day +28 via the replacement of CSA by FK506, and this patient was in stable condition in 2 years after being discharged. The second patient with refractory seizures since day +60 was diagnosed as positive EBV in his cerebrospinal fluid, and then the patient's condition improved after antiviral therapy. However, drop of blood cells and infection occurred in the third patient with seizures on day +90 of haplo-HSCT, and he refused further treatment and finally died.

\section{Follow-up}

Upon follow-up for a median of 13 months, 5 of the 24 patients $(20.8 \%)$ were dead. The survival rates at 3 and 6 months in all patients were $87.5 \%(21 / 24)$ and $83.3 \%$ (20/24), respectively (Fig. 2). Among the five dead patients, two died as a result of early graft rejection; one died because of rejecting further treatment for reduced blood cell counts and pulmonary infection; the other two patients died due to the uncontrollable grade IV aGVHD, among whom one also suffered from serious pulmonary infection 1 year after haplo-HSCT.

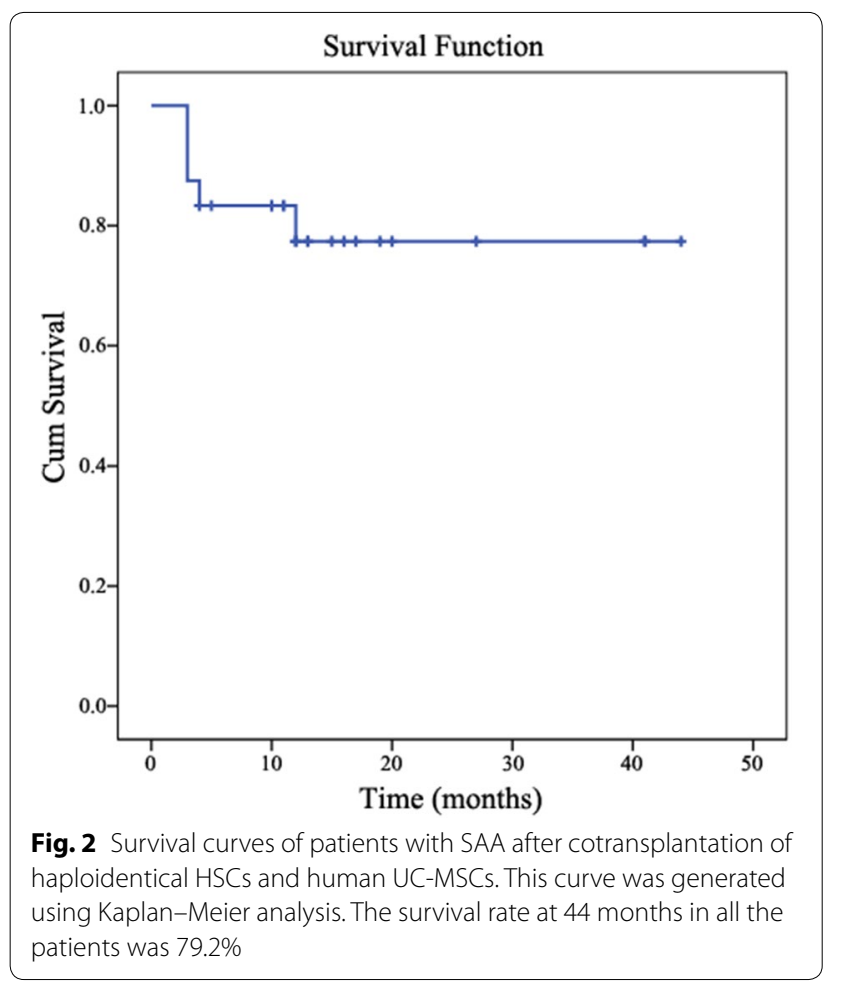

\section{Discussion}

Recent studies suggested that immunosuppressants improved the efficacy of HSCT in patients with SAA [16, 17]. In HLA-matched transplantation for treatment of aplastic anemia, the conditioning regimen consisting of Cy, ATG, and Flu could remarkably decrease the failure rate of transplantation $[18,19]$. Similarly, our study also selected this conditioning regimen in patients with SAA-I; however, we adjusted Cy at the concentration of $500-800 \mathrm{mg} / \mathrm{m}^{2}$ day according to the demographic data of the patients. For patients with SAA-II, reduced doses of $\mathrm{BU}$ was supplemented into the conditioning regimen to enhance immune ablation. Our results demonstrated that all patients with modified conditioning regimen achieved a high engraftment without serious toxic reactions or deaths.

GVHD, as a common complication following allogeneic HSCT, was the principal cause of morbidity and nonrelapse mortality in long-term survivors [20,21]. Our study showed that the incidence of aGVHD after haplo-HSCT was $50 \%$ (six cases of grade I-II aGVHD and six cases of grade III-IV aGVHD), which was consistent with Wu's study showing $57.1 \%$ incidence of aGVHD [3]. However, previous studies showed the incidence ranged of 44-64\% for II-IV aGVHD and 12-26\% for extensive cGVHD [22, 23]. Therefore, it seemed that the infusion of third-party donor-derived UC-MSCs might play a considerable role in reducing the incidence of severe GVHD. Furthermore, our study found that gender, age, donor-recipient relationship, and patient/donor pair were not associated with the incidence of the severe aGVHD, while the incidences of extensive cGVHD were increased in patients with major cross-match mismatch and less HLA loci. Besides, cGVHD incidences were also more likely to occur in males or patients who had developed aGVHD. All these results suggested that it might be an optional prevention route for cGVHD to effectively control severe aGVHD and avoid major cross-match mismatch.

This study showed that the mortality for the entire group of patients was $20.8 \%$, suggesting that cotransplantation of haplo-HSCs and human UC-MSCs might be an effective treatment modality for patients with SAA. However, despite infusions of human UC-MSC after allogeneic HSCT, Si et al. [24] reported that the 3-year overall survival rate was $74.2 \%$, which was lower than our results $(87.5 \%)$. The differences in the survival rate might be attributable to the pediatric patients enrolled in Si et al.s study. Besides, the deaths reported in five patients in this study all occurred due to different complications, such as early graft rejection, reduced blood cell counts, pulmonary infection, and aGVHD, which indicated that preoperative prophylaxis and care also played essential role in a successful HSCT. 
This study has some limitations. First, because cotransplantation of human UC-MSCs and HSCs is not a conventional treatment for patients with SAA, it was difficult to recruit the patients who underwent cotransplantation of human UC-MSCs and HSCs for this study. Due to the small sample size of this study, a prospective study with larger sample size will be performed to confirm our results. Second, a thorough evaluation of the patients to determine the correlation factors affecting GVHD was still required to conduct the cotransplantation. Third, no control group to compare the efficacy and safety of cotransplantation of UC-MSCs and haplo-HSCs was available. Thus, a case-control group comparison of evaluations of treatments with HSCs or UC-MSCs alone is necessary to strengthen the integrity and statistical power of this study and to validate our present results in the future study. Despite these limitations, this study has preliminarily demonstrated the safety and effectiveness of cotransplantation of haplo-HSCs and UC-MSCs for the treatment of patients with SAA.

\section{Conclusion}

This study suggested that the combined application of UC-MSCs in HSCT was a safe and efficacious method for the treatment of SAA. The appropriate conditioning regimen and early treatment for infections and other complications also played a critical role in the success of HSCT.

\section{Additional file}

Additional file 1: Table S1. Pre-transplant characteristics of patients and their donors.

\begin{abstract}
Abbreviations
UC-MSCs: umbilical cord mesenchymal stem cells; SAA: severe aplastic anemia; GVHD: graft versus host disease; HSCT: hematopoietic stem cell transplantation; HLA: the human leukocyte antigen; aGVHD: acute GVHD; CGVHD: chronic GVHD; VSAA: very severe aplastic anemia; CSA: cyclosporine A; G-CSF: granulocyte colony stimulating factor; ATG: anti-human T-lymphocyte immunoglobulin; EPO: erythropoietin; Cy: cyclophosphamide; Flu: fludarabine; MMF: mycophenolate mofetil;TPO: thrombopoietin; PGE1: prostaglandin E1;VOD: veno-occlusive disease; ANC: absolute neutrophil count; STR-PCR: short-tandem repeated sequence-PCR; CMV: cytomegalovirus; EBV: Epstein Barr Virus.
\end{abstract}

\section{Authors' contributions}

$Y W, X Y$, and $Y C$ participated in the design of this study. $X L$ and $B Y$ performed the statistical analysis. LX carried out the study, together with $Z \mathrm{~L}$, and collected important background information. SL and WD helped to draft the manuscript. XW conceived of this study, and participated in the design, and helped to draft the manuscript. All the authors read and approved the final manuscript.

\section{Author details}

1 Department of Hematology, The First Affiliated Hospital of Chinese PLA General Hospital, No. 51 Fucheng Road, Haidian District, Beijing 100048, China 2 Department of Hematology, Chinese PLA General Hospital, Beijing 100853, China.
Acknowledgements

The authors thank everyone in the team for their support.

\section{Competing interests}

The authors declare that they have no competing interests.

\section{Availability of data and materials}

All data generated or analyzed during this study are included in this published article.

Consent for publication

Informed written consents were obtained from all patients.

Ethics approval and consent to participate

The whole protocol was approved by the Institutional Review Board of our hospital and the consents to participate were obtained from all patients.

Funding

This work was supported by The PLA general hospital clinical research support funds (2012FC-TSYS-4007).

\section{Publisher's Note}

Springer Nature remains neutral with regard to jurisdictional claims in published maps and institutional affiliations.

Received: 28 February 2017 Accepted: 20 February 2018

Published online: 01 March 2018

\section{References}

1. Scheinberg P, Young NS. How I treat acquired aplastic anemia. Blood. 2012;120(6):1185-96. https://doi.org/10.1182/blood-2011-12-274019.

2. Wang H, Yan H, Wang Z, Zhu L, Liu J, Guo Z. Cotransplantation of allogeneic mesenchymal and hematopoietic stem cells in children with aplastic anemia. Pediatrics. 2012;129(6):e1612-5.

3. Wu Y, Cao Y, Li X, Xu L, Wang Z, Liu P, et al. Cotransplantation of haploidentical hematopoietic and umbilical cord mesenchymal stem cells for severe aplastic anemia: successful engraftment and mild GVHD. Stem Cell Res. 2014;12(1):132-8.

4. Martelli MF, Di lanni M, Ruggeri L, Pierini A, Falzetti F, Carotti A, et al. "Designed" grafts for HLA-haploidentical stem cell transplantation. Blood. 2014;123(7):967-73

5. Zhu H, Luo RM, Luan Z, Lee V, Zhu YP, Luo CJ, et al. Unmanipulated haploidentical haematopoietic stem cell transplantation for children with severe aplastic anaemia. Br J Haematol. 2016;174(5):799-805.

6. Jaiswal SR, Chatterjee S, Mukherjee S, Ray K, Chakrabarti S. Pre-transplant sirolimus might improve the outcome of haploidentical peripheral blood stem cell transplantation with post-transplant cyclophosphamide for patients with severe aplastic anemia. Bone Marrow Transplant. 2015;50(6):873-5

7. Gupta N, Choudhary D, Sharma SK, Khandelwal V, Dhamija M. Haploidentical hematopoietic SCT for acquired severe aplastic anemia using post-transplant high-dose CY. Bone Marrow Transplant. 2014;50(1):155-6.

8. Yanez R, Lamana ML, García-Castro J, Colmenero I, Ramirez M, Bueren JA. Adipose tissue-derived mesenchymal stem cells have in vivo immunosuppressive properties applicable for the control of the graft-versus-host disease. Stem cells. 2006;24(11):2582-91.

9. Wang HS, Hung SC, Peng ST, Huang CC, Wei HM, Guo YJ, et al. Mesenchymal stem cells in the Wharton's jelly of the human umbilical cord. Stem Cells. 2004:22(7):1330-7.

10. Wu KH, Chan CK, Tsai C, Chang YH, Sieber M, Chiu TH, et al. Effective treatment of severe steroid-resistant acute graft-versus-host disease with umbilical cord-derived mesenchymal stem cells. Transplantation. 2011;91(12):1412-6

11. Wu Y, Wang Z, Cao Y, Xu L, Li X, Liu P, et al. Cotransplantation of haploidentical hematopoietic and umbilical cord mesenchymal stem cells with a myeloablative regimen for refractory/relapsed hematologic malignancy. Ann Hematol. 2013;92(12):1675-84. 
12. Zhang X, Li JY, Cao K, Lu H, Hong M, Qian S, et al. Cotransplantation of HLA-identical mesenchymal stem cells and hematopoietic stem cells in Chinese patients with hematologic diseases. Int J Lab Hematol. 2010;32(2):256-64.

13. Camitta BM, Thomas ED, Nathan DG, Gale RP, Kopecky KJ, Rappeport JM et al. A prospective study of androgens and bone marrow transplantation for treatment of severe aplastic anemia. Blood. 1979;53(3):504-14.

14. Glucksberg H, Storb R, Fefer A, Buckner C, Neiman P, Clift R, et al. Clinical manifestations of graft-versus-host disease in human recipients of marrow from HI-A-matched sibling donor S. Transplantation. 1974:18(4):295-304

15. Xu LX, Cao YB, Wang ZH, Liu ZY, Liu B, Zhao DD, et al. Efficacy of haploidentical allogeneic bone marrow hematopoietic stem cell transplantation combined with umbilical cord blood derived mesenchymal stem cells for severe aplastic anemia. Zhongguo Shi Yan Xue Ye Xue Za Zhi. 2011;19(5):1241-5.

16. Shin $\mathrm{SH}$, Yoon JH, Yahng SA, Lee SE, Cho BS, Eom KS, et al. The efficacy of rabbit antithymocyte globulin with cyclosporine in comparison to horse antithymocyte globulin as a first-line treatment in adult patients with severe aplastic anemia: a single-center retrospective study. Ann Hematol. 2013;92(6):817-24.

17. Maury S, Aljurf M. Management of adult patients older than 40 years refractory to at least one immunosuppressive course: HLA-identical sibling HSCT using fludarabine-based conditioning. Bone Marrow Transplant. 2013;48(2):196-7.

18. George B, Mathews V, Viswabandya A, Kavitha M, Srivastava A, Chandy M. Fludarabine and cyclophosphamide based reduced intensity conditioning $(\mathrm{RIC})$ regimens reduce rejection and improve outcome in Indian patients undergoing allogeneic stem cell transplantation for severe aplastic anemia. Bone Marrow Transplant. 2007;40(1):13-8.

19. Maury S, Bacigalupo A, Anderlini P, Aljurf M, Marsh J, Socié G, et al. Improved outcome of patients older than 30 years receiving HLAidentical sibling hematopoietic stem cell transplantation for severe acquired aplastic anemia using fludarabine-based conditioning: a comparison with conventional conditioning regimen. Haematologica. 2009;94(9):1312-5.

20. Hale G, Jacobs P, Wood L, Fibbe W, Barge R, Novitzky N, et al. CD52 antibodies for prevention of graft-versus-host disease and graft rejection following transplantation of allogeneic peripheral blood stem cells. Bone Marrow Transplant. 2000;26(1):69-76.

21. Okamoto M, Okano A, Akamatsu S, Ashihara E, Inaba T, Takenaka H, et al. Rituximab is effective for steroid-refractory sclerodermatous chronic graft-versus-host disease. Leukemia. 2005;20(1):172-3.

22. Bensinger WI, Martin PJ, Storer B, Clift R, Forman SJ, Negrin R, et al. Transplantation of bone marrow as compared with peripheral-blood cells from HLA-identical relatives in patients with hematologic cancers. N Engl J Med. 2001;344(3):175-81.

23. Couban S, Simpson DR, Barnett MJ, Bredeson C, Hubesch L, Howson-Jan $\mathrm{K}$, et al. A randomized multicenter comparison of bone marrow and peripheral blood in recipients of matched sibling allogeneic transplants for myeloid malignancies. Blood. 2002;100(5):1525-31.

24. Si Y, Yang K, Qin M, Zhang C, Du Z, Zhang X, et al. Efficacy and safety of human umbilical cord derived mesenchymal stem cell therapy in children with severe aplastic anemia following allogeneic hematopoietic stem cell transplantation: a retrospective case series of 37 patients. Pediatr Hematol Oncol. 2014;31(1):39-49.

\section{Submit your next manuscript to BioMed Central and we will help you at every step:}

- We accept pre-submission inquiries

- Our selector tool helps you to find the most relevant journal

- We provide round the clock customer support

- Convenient online submission

- Thorough peer review

- Inclusion in PubMed and all major indexing services

- Maximum visibility for your research

Submit your manuscript at www.biomedcentral.com/submit

C) BioMed Central 